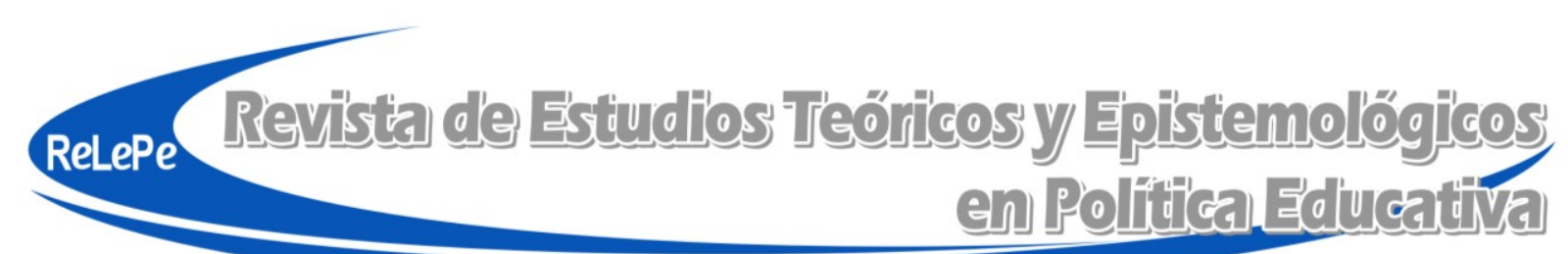

ISSN 2409-3696

DOI: $10.5212 /$ retepe.v.2.018

\title{
La tensión o dilema entre los propósitos de la investigación educativa y del estudio de las políticas educacionales
}

\author{
Jaime Moreles Vázquez \\ Universidad de Colima, México \\ jamovaz@hotmail.com
}

\begin{abstract}
Resumen: En el presente artículo se discute la tensión entre los propósitos de la investigación en los campos de la investigación educativa y el estudio de las políticas de educación. La tensión consiste en las dudas sobre si la investigación culmina cuando es posible hacer una contribución al campo del conocimiento, o concluye cuando los hallazgos generados en un proyecto influyen o tienen algún impacto o repercusión en las prácticas y políticas correspondientes. La aplicación del conocimiento científico es una cuestión relevante desde el origen de esos campos, y, de alguna manera, ha contribuido a configurar la tensión entre los propósitos de la investigación que en ellos se realiza. El dilema o la tensión genera al menos dos tipos de racionalidades: disciplinarias-epistémicas y prácticas-seculares, aunque éstas puedan superponerse o se puede privilegiar una en detrimento de otra. En este trabajo las dos racionalidades se utilizan como extremos para la discusión del tópico.
\end{abstract}

Palabras clave: Investigación Educativa. Políticas Educacionales. Racionalidades.

\section{The tension or dilemma between the purposes of educational research and the study of} education policies

\begin{abstract}
This paper discusses the tension or dilemma between research's purposes in the fields of educational research and the study of education policies. Such tension consists of doubts about whether research culminates when it is possible to make a contribution to the field of knowledge, or concludes when the findings influence or have an impact on practices and policies. Application of scientific knowledge is a relevant issue from the origin of these fields, and it has somehow contributed to configure the tension between the research's purposes. The dilemma or tension generates at least two types of rationalities: disciplinary-epistemic and practical-secular, although these may overlap or one may be favored to the detriment of the other. In this paper the two rationalities are used as extremes to discuss this relevant topic.
\end{abstract}

Keywords: Educational Research. Educational Policy. Rationalities.

\section{A tensão ou dilema entre os propósitos da pesquisa educacional e do estudo das políticas educacionais}

Resumo: Este artigo discute a tensão entre os propósitos da pesquisa nos campos da pesquisa educacional e o estudo das políticas de educação. A tensão consiste nas dúvidas sobre se a pesquisa culmina quando é possível fazer uma contribuição ao campo de conhecimento ou conclui quando os achados gerados em um projeto influenciam ou têm algum impacto ou repercussão nas práticas e políticas correspondentes. A aplicação do conhecimento científico é uma questão relevante da origem desses campos e, de alguma forma, ajudou a moldar a tensão entre os propósitos da pesquisa realizada neles. $\mathrm{O}$ 
dilema ou a tensão geram pelo menos dois tipos de racionalidades: práticas disciplinares-epistêmicas e seculares, embora estas possam se sobrepor ou se possa privilegiar uma em detrimento de outra. Neste trabalho, as duas racionalidades são usadas como fins para a discussão do tópico.

Palavras chave: Pesquisa educacional. Políticas educacionais. Racionalidades.

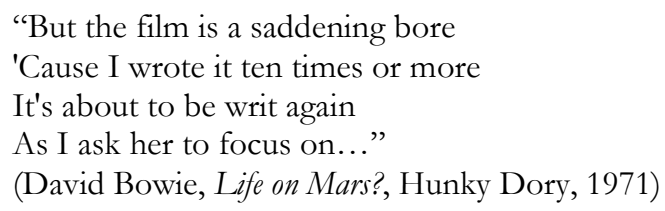

\section{Distinciones conceptuales y premisas}

En las prácticas de investigación intervienen elementos más allá de los puramente epistémicos. Aquí empleamos algunos de éstos para explorar el dilema o tensión existente entre los propósitos científicos, tanto del estudio de las políticas educacionales como de la investigación que se realiza en el campo educativo, investigación en o para (en o de) las políticas o la educación, según corresponda. Argumentamos sobre esa tensión desde las racionalidades mediante las cuales comprendemos y fundamentamos nuestras actividades científicas, y que nos llevan a decidir entre distintos cursos de acción.

Podemos conjeturar que el dilema o tensión entre los propósitos científicos existe desde el origen de los campos de la educación y del estudio de las políticas educacionales, pues, además del desarrollo disciplinario, también se ha pretendido contribuir al hecho educativo y a la vida democrática, respectivamente (Heck, 2004; Sandín, 2003; Ungerleider, 2012). En ese sentido, la investigación que se hace en el campo educativo a menudo se asocia con propuestas y recomendaciones sobre lo que se investiga y a su potencial utilización en las prácticas a las que se refiere (Heck, 2004).

Con el paso del tiempo, la búsqueda de la influencia en las prácticas y las políticas se ha ido diluyendo, principalmente por el predominio del código disciplinario en la ciencia actual (Fenwick \& Farrel, 2012; Wesseley, 1996), y también debido a los sistemas de promoción y recompensas del trabajo científico (Lawrence, 2008; Stephan, 2012). Tales sistemas desincentivan la interacción entre la investigación y las prácticas (Mitton et al., 2007, citado en Levin \& Cooper, 2012) y generan una tensión entre la búsqueda de conocimientos para la intervención social y para el debate al interior de un campo especializado. De acuerdo con algunos autores, como investigadores no hemos perdido nuestra doble vocación intelectual de intervención y de compromiso político, porque creemos que nuestro trabajo tiene valor práctico y lo consideramos relevante para los demás actores sociales (Contreras, 1996; Miller et al., 2011).

Desde esos planteamientos nutrimos nuestra conjetura sobre la tensión o dilema entre los propósitos científicos que ha acompañado a las ciencias sociales en general, y a los estudios de la educación y de las políticas educacionales en particular. También de ahí proponemos las racionalidades como ejes de discusión, pues explicarían nuestra posición sobre el alcance o propósito final del trabajo de investigación, la publicación científica, la formación personal, la repercusión o influencia en el ámbito científico y/o la influencia en la práctica y la toma de decisiones.

Las racionalidades son observables en las prácticas de la investigación, y por esa razón se construyen desde el razonamiento de los científicos sobre su propio trabajo (Mbock et al., 2004). 
Pensamos que pueden ayudarnos a comprender el dilema o tensión, ya que nos permiten concebir y justificar las actividades que realizamos, así como construir las visiones y perspectivas que otorgamos a esas prácticas para dotarlas de sentido. Argüimos que tales nociones se construyen en función de la socialización disciplinaria y de las características de las comunidades científicas de referencia, aunque son matizadas también por la forma como se regulan e incentivan las actividades científicas en un contexto determinado.

La tensión o dilema también se vincula con decisiones u omisiones muy relevantes para las prácticas de investigación individuales o grupales, pues a éstas se circunscriben, entre otras cuestiones, la elección de problemas u objetos de estudio, de procedimientos metodológicos, de referentes interpretativos y medios de difusión o divulgación, la construcción de redes de colaboración, y, principalmente, la elección de sus metas o propósitos finales, que es el eje de nuestra argumentación.

Asumimos la investigación como una actividad sistemática y rigurosa que genera conocimientos, saberes e insumos de información de interés principalmente disciplinario, y, en ocasiones, de interés práctico. Esto a pesar de que el compromiso primordial de la investigación es precisamente con las disciplinas, es decir, contribuir a un cuerpo de conocimientos o generar entendimiento sobre ciertos temas-fenómenos, de acuerdo con algunas referencias (Davies \& Nutley, 2008; Foreman-Peck \& Winch, 2010).

Cuando aludimos a lo disciplinario, nos referimos a que los saberes generados amplían el conocimiento sobre determinado asunto de interés científico, además de contribuir a la agenda de investigación correspondiente. Por el contrario, cuando hablamos de cuestiones de interés práctico, aludimos a los insumos de información que, aunque se generan de actividades científicas, se van constituyendo como marcos de referencia para las actividades cotidianas, saberes que contribuyen a la comprensión de las prácticas, e incluso ayudan a orientarlas.

Como antes mencionábamos, argüimos que las implicaciones disciplinarias o prácticas de nuestras actividades científicas, y que están vinculadas a la tensión o dilema entre sus propósitos, se pueden entender desde las racionalidades que tomamos como marcos de referencia para la discusión: racionalidad disciplinaria o epistémica y racionalidad práctica o secular; la distinción epistémica y secular fue propuesta por Kogan (2006). Es preciso reiterar que en el documento sólo nos referimos a los propósitos o metas finales de la investigación y asumimos las racionalidades como puntos de referencia, sin ignorar que entre una y otra existen gradaciones.

En ese sentido, la presente discusión se enmarca en la tradición de los estudios del uso de la investigación, pues la potencial aplicación del conocimiento es uno de los extremos de la tensión, investigación para, aunque no en el sentido estricto del término, pues la idea de uso instrumental de la investigación en la política y en la práctica ha sido abandonada desde hace ya varios años por casi todos los interesados en el tema (Fenwick \& Farrel, 2012; Saunders, 2007).

Los estudios del uso de la investigación han mostrado que éste es indirecto y sutil, y que se concreta en el mediano y largo plazo. Se trata de un tipo de uso que se denomina iluminativo o conceptual, pues los hallazgos de la investigación van filtrándose gradualmente en los círculos del debate público y político, así como en las prácticas correspondientes. Se trata de una clase de utilización del conocimiento científico que lleva a los actores a pensar diferente o más ampliamente acerca de alguna problemática o situación (Fenwick \& Farrel, 2012; Levin \& Cooper, 2012). Del uso conceptual a lo que se concibe como uso instrumental hay un trecho amplio; también hay otras modalidades del uso, aunque no son de interés para la presente argumentación. 
Por tanto, como representa una de las partes del dilema que estamos discutiendo, investigación para las políticas educacionales y para la educación, pensamos que es pertinente dejar en claro la complejidad y las implicaciones que conlleva el uso, es decir que se concatenen circunstancias y factores para que efectivamente la investigación concrete sus implicaciones prácticas. En lo que sigue argumentamos cómo está constituida la tensión o dilema entre propósitos científicos y racionalidades, para después hablar del compromiso político de la investigación y plantear algunas conclusiones a manera de agenda de discusión.

\section{La tensión o dilema entre propósitos científicos}

La discusión sobre la tensión o dilema entre los propósitos de las actividades científicas es un tema pertinente. Tanto en el campo de la educación como en el de las ciencias políticas, la potencial aplicación del conocimiento ha representado un asunto de interés. Mientras que en el campo de la educación se ha mantenido como un tema de actualidad (Fenwick \& Farrell, 2012), en el de las ciencias políticas ha sido enlistado en una serie de Big Things para la agenda de las disciplinas implicadas (Goodin, 2011). Pensamos que este tipo de debates constituyen agendas de reflexión que fortalecen las disciplinas, pues la discusión teórica implica siempre la discusión de la práctica, de acuerdo con algunas referencias (Berndtson, 2010; Heck, 2004; Thoenig, 2004).

En ese sentido, partimos de la premisa respecto a que en las disciplinas implicadas está siempre latente la cuestión acerca de si los procesos de investigación culminan cuando se logra una mejor explicación o comprensión de los fenómenos estudiados, o bien, finalizan cuando influyen o acontecen algunas repercusiones en las prácticas y políticas relacionadas, aunque este tipo de efectos sean muy difíciles de evaluar y/o valorar, según Davies y Nutley (2008).

Como anticipábamos unas páginas atrás, la tensión o dilema entre los propósitos científicos ha estado presente desde el origen de los campos en cuestión, inscribiéndose en las tradiciones de investigación que se han venido desarrollando hasta la actualidad. Las raíces de la investigación sobre las políticas educacionales se hallan en las ciencias políticas (Heck, 2004), mientras que las de la investigación en el campo de la educación se encuentran en la pedagogía experimental (Sandín, 2003).

En el primer caso se buscaba que la investigación contribuyera a la administración pública, y que, de esa manera, coadyuvara a que se hicieran mejores políticas y se fortaleciera la vida democrática. En el segundo caso, se proponían estudios que tuvieran repercusiones en la práctica escolar, es decir, la aplicación del conocimiento científico en el aula para la mejora del aprendizaje y del hecho educativo en general.

Lo anterior no quiere decir que esas tradiciones se hayan mantenido con el paso de los años, ni tampoco que quienes nos formamos y nos dedicamos profesionalmente a cultivar tales disciplinas seamos conscientes de ello. No obstante, queremos resaltar que la aplicación del conocimiento derivado de las prácticas de investigación estaba en el espíritu de las actividades de esos campos. Paulatinamente, con la disciplinarización y la profesionalización de las actividades científicas, esas raíces se habrían ido diluyendo, dando paso, entre otras cuestiones, al dilema o tensión que aquí hemos propuesto como eje argumentativo.

Por disciplinarización entendemos el proceso por el que han pasado las diferentes ciencias, desde que se practicaban casi como actividades artesanales, hasta constituirse en actividades profesionales. Las disciplinas científicas, las ciencias en general, replican las tres etapas de la historia de la ciencia moderna: institucionalización, profesionalización, industrialización 
(Berndtson, 2010; Kreimer, 2009). Por su naturaleza, quizás la investigación de la educación y la de las políticas educacionales permanezcan en la profesionalización.

La profesionalización de una disciplina científica implica la creación de instituciones, la formación de organismos y asociaciones, la instauración de mecanismos de comunicación y el desarrollo de programas mediante los que se reconocen, regulan e incentivan las actividades científicas, y que constituyen la identidad de las diferentes áreas del conocimiento (Berndtson, 2010). Los mecanismos de control, evaluación e incentivación, además de promover el desarrollo científico, actúan como constreñimientos de las prácticas en las cuestiones relativas a la aplicación del conocimiento científico, o la consecución de uno de los propósitos en tensión.

En ese sentido, el dilema entre lo disciplinario y lo práctico ha potenciado elementos obstaculizadores, como la falta de identidad intelectual y de integración de conocimientos y prácticas (Johnson, 2003, citado en Heck, 2004), ya que, en comparación con áreas como la salud y otras disciplinas de las ciencias naturales, el estudio de la educación y de las políticas educacionales han sido tipificados como campos fragmentados y desorganizados (Levin \& Cooper, 2012). Algunas referencias incluso sugieren que se trata de campos marginales y de poco interés, en los que, debido al aislamiento de individuos y grupos, cada quien puede investigar lo que quiera (De la Orden y Mafokozi, 1999; Enders, 2010; Jakobi et al., 2010).

También podemos considerar el hecho de que ambos campos poseen objetos de estudio multidisciplinarios. Aunque esto puede interpretarse como una fortaleza, por el componente heurístico que conlleva, nos interesa resaltar los desafíos que representa para la conjunción de las prácticas científicas. Esto ha sido aún más visible en la tradición de la investigación sobre políticas de la educación en los años recientes, que ha fungido como piedra angular para la interacción de diferentes disciplinas: evaluación de políticas, en donde la economía y la sociología se traslapan; estudios de la educación acerca de los temas de democracia y gobierno, conjugando el derecho y las disciplinas jurídicas con la filosofía política, la historia y las ciencias de la educación; y, finalmente, investigación que busca iluminar o informar el proceso de la toma de decisiones y la hechura de políticas, principalmente en los temas de privatización, internacionalización, reforma de los sistemas educativos, nuevos modelos de gobernanza, entre otros (Enders, 2010; Jakobi et al., 2010; Opfer et al., 2008; Heck, 2004).

En ese sentido, siguiendo a Fenwick y Farrell (2012), podemos argüir que la investigación de la educación y los estudios de políticas parten de marcos referenciales diversos, se refieren a modelos indefinidos y sin límites claros, y que a menudo implican conceptos y terminologías difusas. La falta de certidumbre respecto a la operatividad de modelos y proposiciones teóricas genera cuestionamientos sobre las prácticas que se realizan. De acuerdo con Sartori (2012), esto ocurre con las ciencias sociales en general, pues es frecuente que los modelos teóricos se traslapen, se contradigan, e incluso que generen estiramientos conceptuales que, aunque otorgan amplitud explicativa, demeritan la precisión connotativa, que es justamente lo que consolida el estatus de cientificidad de una disciplina, la precisión del lenguaje. La falta de precisión quizás también disminuya las posibilidades de que una disciplina trascienda la profesionalización, según las etapas que mencionábamos antes, y que además limite las posibilidades de aplicación de la investigación, que representa una de las modalidades de los propósitos científicos que venimos discutiendo.

Pensamos que las racionalidades que guían las prácticas científicas replicarían la falta de cohesión de los campos o áreas de referencia. Y en el caso de la tensión o dilema entre los propósitos científicos, existirían distintas variaciones: el predominio de una racionalidad sobre otra, la coexistencia de ambas, posiciones híbridas en donde una racionalidad prevalece, e incluso una versión en donde la predilección por una de ellas implica la denostación de la 
opuesta. Es probable que también exista cierto soslayamiento de estas cuestiones y que, en ocasiones, la que se declara contradiga a la que se practica.

\section{Las racionalidades}

Las racionalidades se adquieren en los procesos de aculturación de las disciplinas, en la socialización disciplinaria, se van fortaleciendo en las prácticas y en el trabajo cotidiano, y son moldeadas por los programas de regulación e incentivación de las actividades científicas. También por el sentido de pertenencia que los grupos dan a los individuos, pues son las comunidades y grupos los que asignan, determinan y regulan los problemas y las estrategias de investigación de sus integrantes (Bourdieu, 2005; Merton, 2002). Como es mayor el peso de las características de las comunidades que el de cada uno de sus integrantes, son las comunidades las que influyen y dan forma al dilema entre los propósitos científicos; en esto parece no intervenir en gran medida lo que apuntábamos antes respecto a la fragmentación de los campos de la investigación de la educación y del estudio de las políticas educacionales.

Las variaciones entre las diferentes comunidades implicarían una jerarquización distinta de la escala de los valores que les dan forma y contenido (Becher, 2001). En este caso, respecto a la tensión entre los propósitos científicos, aludimos a los valores que subyacen a que las actividades tiendan hacia la orientación de las prácticas correspondientes, o bien, valores que conduzcan la investigación hacia el fortalecimiento de las disciplinas de referencia.

Los valores disciplinarios se adquieren y refuerzan en la formación dentro de los programas escolares y en las instituciones educativas, a diferencia de los valores prácticos que se fortalecen mediante la socialización profesional y los contextos laborales; por esas razones hay disciplinas y comunidades más orientadas por valores epistémicos y otras por valores extraídos de la pertinencia social de la investigación que realizan (Allen et al., 2007; Becher, 2001).

En esto también influyen, entre otros factores, el grado de consolidación y el capital científico del campo en cuestión, pues las disciplinas más consolidadas son un tanto más autónomas respecto a la influencia de factores externos y a menudo se rigen primordialmente por criterios determinados internamente, a diferencia de lo que ocurre con las disciplinas menos consolidadas (Bourdieu, 2005). Aunque también es un poco desconcertante que disciplinas con avance o desarrollo precario sean autónomas a factores externos, quizás por la indiferencia que hacia ellas tienen, por ejemplo, sectores de la iniciativa privada y hasta los organismos y las dependencias públicas que financian la investigación científica.

Las disciplinas que tienen como objeto de estudio a la educación y a las políticas educacionales combinan valores académicos y profesionales, además de que a menudo son foco de la influencia de factores externos; esto ocurre a pesar de la escasa pertinencia social de sus actividades científicas. De acuerdo con lo anterior, en la investigación que se realiza en esos campos se traslapan las metas o propósitos científicos, los intereses disciplinarios y prácticos, y las distintas racionalidades.

Con base en lo que venimos planteando, vale la pena reiterar que la distinción que hacemos entre los dos tipos de racionalidades, disciplinaria-epistémica y práctica-secular, es ante todo con propósitos analíticos, pues no se trata de un asunto en el que los polos o extremos sean evidentes, sino que más bien en nuestra argumentación constituyen puntos de referencia que nos permiten el análisis del dilema entre los propósitos científicos en las prácticas de investigación de los campos de la educación y de las políticas educacionales. 
En la racionalidad disciplinaria o epistémica el dilema o tensión de los propósitos científicos se inclina hacia la búsqueda del fortalecimiento del campo en cuestión. A partir de esta racionalidad, el propósito de la investigación es generar conocimiento, siguiendo el modo tradicional de producción, que implica su difusión mediante canales especializados y con un lenguaje hasta cierto punto abstracto, o al menos en el código de la disciplina de referencia; es decir, se privilegian los mecanismos habituales de la discusión entre pares, revistas especializadas, libros, congresos, entre otros. En ese sentido, el trabajo que se realiza desde esta racionalidad se desliga de las políticas y las prácticas educativas, pues sus procedimientos son independientes de su aplicación y su fin último se concreta cuando se generan nuevos conocimientos (Davies \& Nutley, 2008; Holbrook et al., 2000).

Durante los procesos de formación, este tipo de valores y concepciones sobre la práctica científica y sus finalidades son imbuidas, compartidas y debatidas entre quienes poseen más experiencia y los novatos o aprendices que quieren hacerse parte de cierta comunidad, pues es mediante el trabajo disciplinario como éstos son socializados en las prácticas y los nuevos problemas son legitimados (Berndtson, 2010). El objetivo de estos procesos es que tales significaciones se constituyan como racionalidades de las actividades científicas, al grado de normalizarlas y que ya no sean parte de la discusión de las comunidades o grupos.

En este caso estamos hablando de una jerarquización de valores epistémicos o disciplinarios en las que se privilegia el acatamiento de las normas que actualmente rigen a la ciencia: el factor de impacto (FI), el indice $h$, la publicación como la meta final de casi todo proceso de investigación, es decir, el fetiche de los peer-reviewed articles, que aparentemente representan la muestra más estandarizada o universalizada de la ciencia (Schmitter, 2002, citado en Berndtson, 2010; Stephan, 2012).

La preeminencia del paper ha permeado a tal grado el trabajo científico actual, que desde hace algunos años se habla de disciplinas basadas en artículos (Goodin, 2011); aunque quizás esta característica es atribuible apenas a algunas de las ciencias sociales, pues las humanidades y la educación estarían basadas en libros o capitulos de libros (Giménez, 2014). En cualquiera de ese tipo de publicaciones, estamos ante la cuestión de que el trabajo se ciña y se valore casi exclusivamente por los productos publicados (Miller et al, 2011).

Cuando el paper constituye el criterio por antonomasia de las comunidades científicas, se iría construyendo una racionalidad que entre sus planteamientos y premisas lleve a denostar a la racionalidad práctica, arguyendo que pretender la aplicación del conocimiento contamina el proceso de investigación (Levin, 2004; Shinn, 2007), y que salirse de la disciplina actúa en contra de las propias comunidades científicas (Fenwick \& Farrell, 2012), pues esos propósitos distraen de las verdaderas preocupaciones de la ciencia y de los problemas legítimos. Poner en segundo término o soslayar las implicaciones prácticas de las actividades científicas suele ser también una de las causas de la fragmentación de grupos o comunidades. Y la fragmentación es una de las características de la investigación de la educación y del estudio de las políticas educacionales, como ya habíamos mencionado antes.

\section{Racionalidad práctica o secular}

La investigación educativa y el estudio de las políticas educacionales son un campo principalmente aplicado, de acuerdo con Heck (2004). Desde el inicio la mayoría de los proyectos la investigación se comprometen a generar recomendaciones para las prácticas y las políticas, y a que 
sus resultados sean un insumo para la toma de decisiones; generar lo que se denomina como conocimiento para la acción (Graham et al., 2006, citados en Fenwick \& Farrel, 2012).

En el estudio de las políticas, incluso en algunos trabajos se hacen corresponder las fases de la política con algunas de las modalidades de utilización potencial de la investigación propuestas por Carol Weiss. Problem solving correspondería con implementing, la investigación clarifica las opciones de política. Political (deciding): la investigación sustenta una decisión o medida previamente tomada. Enlightement (designing): la investigación provee de ideas generales y de conceptualizaciones de determinados problemas (Klemperer et al., 2003).

En la racionalidad práctica o secular se apela a la idea de la ciencia como una actividad de beneficio social, que representa uno de los supuestos arraigados en el imaginario de las comunidades científicas, y también de otros grupos y sectores sociales. La noción del bien común como marco de referencia, una pretensión de que el trabajo científico tenga posibilidades de impacto en contextos más allá de lo académico (Holbrook et al., 2000, Figgis et al., 2000).

En ese sentido, en esta racionalidad el dilema o tensión de los propósitos científicos se inclina hacia la búsqueda de que la investigación influya en las situaciones o problemáticas a las que se refiere. Se pretende producir conocimientos útiles para la resolución de los problemas que enfrentan los sistemas educativos y hasta para el cambio de las políticas en la materia. La investigación se concibe como una actividad orientada por sus implicaciones prácticas (Davies \& Nutley, 2008; Holbrook et al., 2000) y como un potencial instrumento de cambio (Carr \& Kemmis, 1986; Whyte, 1991, citados en Allen et al., 2007). Así ocurre también en algunas de las áreas comprendidas en los campos de la salud, el trabajo social y el derecho (Becher, 2001). Esto no quiere decir que la influencia de los resultados de la investigación se corresponda con las expectativas que se han generado al respecto.

Aunque de antemano se asume que los investigadores no hacen esfuerzos suficientes para relacionarse con grupos más allá del ámbito académico (Edwards et al., 2007, citado en Fenwick \& Farrell, 2012), quienes promueven esta racionalidad critican la inacción que existe entre los grupos científicos que se orientan por criterios puramente epistémicos. Esto debido a que la investigación que se realiza se le considera una actividad científica comprometida, por las implicaciones que conlleva para las prácticas (Flyvberj, 2001, citado en Edwards, 2012). Actividades que adquieren sentido al orientarse hacia el mejoramiento de la política y de la acción educativa (Bassey, 1999, citado en Foreman-Peck \& Winch, 2010).

Siguiendo estas premisas, existen investigadores que se tornan en una suerte de paladines de la investigación (Carden, 2004; Puchner, 2003; Stacki, 2003), ya que además de estudiar temas política o socialmente relevantes, implican a otros actores en el proceso de investigación y emplean modalidades alternativas de difusión. Asimismo, establecen nexos con actores que pretenden que la investigación sea un marco de referencia para sus prácticas, y procuran cultivarlos (Davies \& Nutley, 2008).

\section{Cierre o agenda: el tenue compromiso de la investigación con las políticas y las prácticas}

Como apuntábamos antes, la distinción entre las racionalidades disciplinaria-epistémica y práctica-secular la planteamos en un sentido analítico. Ambos extremos constituyen puntos de referencia para mostrar la tensión o dilema existente entre los propósitos o metas de la investigación que se realiza en los campos de la educación y del estudio de las políticas educacionales. Por las características de esos campos, lo más probable es que tales racionalidades 
convivan en los diferentes grupos y comunidades científicas que integran esas disciplinas, evidenciando coherencias y contradicciones de las prácticas.

La investigación de la educación está política e ideológicamente comprometida, al grado de que algunas referencias la denominan como la ciencia social comprometida (Foreman-Peck \& Winch, 2010; Rickinson et al., 2011). Por su parte, en el campo de las políticas educacionales ocurre algo similar, pues todo lo que se estudia es potencialmente político, como lo muestra la mayoría de los temas que constituyen las principales políticas sociales: desempleo, salud, pobreza, equidad, desarrollo económico (Macridis, 1968, citado en Sartori, 2012).

$\mathrm{Al}$ representar una actividad basada en valores, la práctica científica constituye un acicate para las creencias y significaciones personales. Así es como nos enfrentamos al dilema o tensión de sus propósitos: contribuir a la toma de decisiones y a la hechura de políticas, o bien, tomar distancia de ese tipo de cuestiones centrándose en buscar aportaciones para el desarrollo disciplinario o epistémico.

La tensión o dilema entre los propósitos obedece al hecho de que una buena parte de los estudios que se llevan a cabo se originan en las prácticas cotidianas, y también al compromiso e implicaciones políticas que subyacen a las actividades que se realizan en esos campos. Se supone que el origen de los problemas, de alguna manera, determinaría el alcance de los resultados, a quién estarían dirigidos y por qué, lo que comienza en la práctica regresaría a la práctica, lo que comienza en las disciplinas, a éstas retornaría (Coleman, 1972, citado en Heck, 2004). Pero en la investigación de la educación y del estudio de las políticas, no hay retorno garantizado del conocimiento a las prácticas o al debate sobre las políticas. En nuestro trabajo, los académicos podemos o no comprometernos con la acción social, y a veces incluso evitamos cuestiones de controversia, manteniéndonos en el sigilo del laboratorio o del cubículo, según Becher (2001).

De acuerdo con esos planteamientos, en nuestras actividades científicas podríamos desinteresarnos del debate público y de temas potencialmente políticos, o en sentido inverso, abiertamente enfocarnos en ellos y hasta tomar partido. Esto porque todo conocimiento es inevitablemente político, pues sirve a los intereses de ciertos individuos y grupos y se opone a los intereses de otros, con base en lo señalado por algunos autores (Donmoyer, 1995, citado en Ginsburg y Gorostiaga, 2003; Mbock et al., 2004).

Otra vez, la toma de postura o partido la planteamos a partir de dos polos a modo de puntos de referencia, basándonos en la propuesta de Gewirtz (2003), accomodation y disruption, que representarían las posiciones que asume la investigación respecto al discurso gubernamental u oficial, o respecto a temas políticos o acerca de iniciativas específicas. En la primera postura la investigación se acomoda al punto de promover los discursos oficiales, mientras que en la segunda la investigación rompe o al menos pretende ser un contrapeso respecto a posiciones oficialistas.

La investigación políticamente comprometida pretendería poner a prueba la validez de las políticas oficiales, elevando la calidad del debate público sobre temas de interés general (Biao \& Shen, 2005; Florence \& Martiniello, 2005), o bien, legitimándolas a través de la generación de ideas y discursos que cedan a los intereses de la política, investigación que legitima decisiones o acciones compatibles con intereses políticos o personales (Weiss, 1979), y que justifica la organización del poder (Mills, 1961). En las ciencias políticas, esta visión es concebida como la sumisión o derrota del conocimiento ante la política (Torgerson, 1992).

La meta final de la investigación de la educación y de las políticas educacionales es el cambio educativo y también el cambio político, pues la investigación es de naturaleza política, es otra manera de hacer política, una forma de echar a andar un conjunto de valores y posiciones 
sobre los temas o problemas que nos parecen relevantes. En ese sentido, la tensión o dilema que se desprende de esas características nos representa una versión de la tesis marxista sobre interpretar o cambiar el mundo (Goodin, 2011), pues nos resulta un tanto paradójico que una buena parte de la investigación se desentienda de otros propósitos más allá de los epistémicos, a pesar de que casi cualquiera de sus procesos se originen en problemas de la práctica educativa y social.

No tratamos de renegar del compromiso disciplinario que toda actividad científica posee, sino más bien de problematizar la cuestión. Orientar la investigación mediante racionalidades disciplinarias no ha representado una mala alternativa, pero ésta no es la única vía. Tanto la investigación de la educación como aquella sobre política educativa sí poseen compromiso social, puesto que pueden contribuir a la democracia. Como mencionaba Dewey (2004) sobre la educación, ¿la investigación puede ayudar a hacer una sociedad más democrática?

Por esas razones son muy relevantes las racionalidades, pues están construidas desde los fundamentos epistemológicos que nos guían. Ahí radica igualmente la pertinencia de los estudios epistemológicos propuestos por Tello y Mainardes (2012). También es crucial la conciencia que tenemos sobre estas cuestiones, pues podemos no atribuirles el valor que corresponde e, incluso, actuar epistemológicamente a ciegas, o hasta dolosamente a ciegas, lo que representa uno de los excesos o efectos no deseados del dilema o tensión entre los propósitos científicos, ya que implicaría no declarar nuestra postura u ocultarla, o no tomar postura, a sabiendas de que nuestro trabajo pueda utilizarse para legitimar ciertas posiciones políticas o iniciativas precisas. Es importante, al menos, declarar los principios e intereses desde los que se parte. Una suerte de declaración inicial, como se está solicitando en algunas convocatorias a quienes estudiamos temas socialmente relevantes.

\section{Referencias}

Allen, P., Peckham, S., Aderson, S. \& Goodwin, N. (2007). "Commissioning research that is used: the experience of the NHS Service Delivery and Organization Research and Development Program". Evidence \& Policy, 3(1), 119-134.

Becher, T. (2001). Tribus y territorios académicos. La indagación intelectual y las culturas de las disciplinas. Madrid: Gedisa.

Berndtson, E. (2010). "Education policy and the harmonization of political science as a discipline”. In: Kajobi, A. P., Martens, K. \& Dieter Wolf, K. (Eds.). Education in political science. Discovering a neglected field. New York: Routledge-ECPR.

Biao, X. \& Shen, T. (2005). "Does migration matter in China? A review of its relationship to policy since the 1980s". International Journal on Multicultural Societies, 7(1), 11-32.

Bourdieu, P. (2005). Ideología, política y poder (4ª reimpresión). Buenos Aires: EUDEBA.

Carden, F. (2004). "Issues in assessing the policy influence of research". International Social Science Journal, 56(179), 135-151.

Contreras, O. F. (1996). "Las ciencias sociales y la vida real". Estudios sobre las Culturas Contemporáneas, 2(4), 137-149.

Davies, H. T. O. \& Nutley, S.M. (2008). Learning more about how research-based knowledge gets used: guidance in the development of new empirical research. New York: William T. Grant Foundation. 
De la Orden, A. \& Mafokozi, J. (1999). "La investigación educativa: naturaleza, funciones y ambigüedad de sus relaciones con la práctica y la política educativas". Revista de Investigación Educativa, 17(1), 7-29.

Dewey, J. (2004). Democracia y educación. Madrid: Morata.

Edwards, A. (2012). User engagement and the process of educational research. In: T. Fenwick, \& L. Farrell (2012). Knowledge mobilization and educational research. Politics, languages and responsibilities. New York: Routledge.

Enders, J. (2010). Political science and educational research: windows of opportunity for a neglected relationship. In: A. Jakobi, K. Mertens \& K. Dieter (Eds.). Education in political science. Discovering a neglected field. New York: Routledge/EPCR.

Fenwick, T. \& Farrell, L. (2012). Introduction: knowledge mobilization. The new research imperative. In: T. Fenwick \& L. Farrell (Eds.). Knowledge mobilization and educational research. Politics, languages and responsibilities. New York: Routledge.

Figgis, J., Zubrick, A., Butorac, A. \& Alderson, A. (2000). Backtracking practice and policies to research. In: Department of Education, Training \& Youth Affairs (DETYA). The impact of educational research. Australia: DETYA.

Florence, E. \& Martiniello, M (2005). Social Science research and public policies: the case of immigration in Belgium. International Journal on Multicultural Societies, 7(1), 50-67.

Foreman-Peck, L. \& Winch, C. (2010). Using educational research to inform practice. A practical guide to practitioner research in universities and colleges. New York: Routledge.

Gewirtz, S. (2003). Enlightening the research-policy relationship: issues and dilemmas for educational researchers. Paper presented at the European Conference on Educational Research, University of Hamburg, 17-20 September 2003. Recuperado en 14 noviembre, 2004, de http://www.kcl.ac.uk/depsta/education/papers/SGecer.pdf

Giménez, E. (2014). Imposturas en el ecosistema de la publicación científica. Revista de Investigación Educativa, 32(1), 13-23.

Ginsburg, M. \& Gorostiaga, J. (Eds.) (2003). Limitations and Possibilities of Dialogue among Researchers, Policymakers and Practitioners. London: Routledge Falmer.

Goodin, R. (2011). The state of the discipline, the discipline of the state. The Oxford handbook of political science. New York: Oxford University Press.

Heck, R. H. (2004). Studying educational and social policy. theoretical concepts and research methods. New Jersey: LEA.

Holbrook, A., Ainley, J., Bourke, S., Owen, J., McKenzie, P., Misson, S. \& Johnson, T. (2000). Mapping educational research and its impact on Australian schools. In: Department of Education, Training \& Youth Affairs (DETYA). The impact of educational research. Australia: DETYA.

Jakobi, A., Mertens, K., \& Dieter, K. (Eds.) (2010). Education in political science. Discovering a neglected field. New York: Routledge/EPCR. 
Klemperer, A., Theisens, H. \& Kaiser, F. (2003). Dancing in the Dark. The Relationship between Policy Research and Policy Making in Dutch Higher Education. In: M. Ginsburg \& J. Gorostiaga (Eds.). Limitations and possibilities of dialogue among researchers, policymakers and practitioners. London: Routledge Falmer.

Kogan, M. (2006). Knowledge and politics in the use and formation of research. Paper given at Seminar, University of Bergen. 18-19 May 2006. Recuperado en 11 enero, 2007, de http://www.knowpol.uib.no/docs/papers/2006/kogan.pdf

Kreimer, P. (2009). El científico también es un ser bumano. La ciencia bajo la lupa. Buenos Aires: Siglo XXI Editores.

Lawrence, P. (2008). "Lost in publication: how measurement harms science". Ethics in Science and Environmental Politics, 8, 9-11.

Levin, B. (2004). "Making research matter more". Education Policy Analysis Archives, 12(56), 1-20.

Levin, B. \& Cooper, A. (2012). Theory, research and practice in mobilizing research knowledge in education. In: T. Fenwick, \& L. Farrell (Eds.). Knowledge mobilization and educational research. Politics, languages and responsibilities. New York: Routledge.

Mbock, C. G., C., Ngo-Mpeck, M. L., Kom, D. \& Zambo, J. (2004). "Policy utilization of research results in Cameroon". International Social Science Journal, 56(179), 37-45.

Medawar, P. B. (2011). Consejos a un joven cientifico. Barcelona: Crítica.

Merton, R. (2002). Papel del intelectual en la burocracia política. In: Teoría y estructura sociales (4a ed). México: Fondo de Cultura Económica.

Miller, A. N., Taylor, S. G., \& Bedain, A. G. (2011). "Publish or perish: academic life as management faculty live it". Career Development International, 16(5), 422-445.

Mills, W. (1961). La imaginación sociológica. México: FCE.

Opfer, V. D., Young, T. \& Fusarelli, L. (2008). "Politics of interest: interest groups and advocacy coalitions in American education”. In: B. S. Cooper, J. G. Cibulka \& L. D. Fusarelli (Eds.). Handbook of education. Politics and policy. Nueva York: Routledge.

Puchner, L. (2003). "Researching women's literacy in Mali: a case study of dialogue among researchers, practitioners, and policy makers". In: M. Ginsburg \& J. Gorostiaga (Eds.). Limitations and possibilities of dialogue among researchers, policymakers and practitioners. London: Routledge Falmer.

Rickinson, M. Sebba, J. \& Edwards, A. (2011). Improving research trough user engagement. New York: Routledge.

Sandin, M. P. (2003). Investigación cualitativa en educación. Fundamentos y tradiciones. Madrid: McGrawHill.

Sartori, G. (2012). La Política. Lógica y método en las ciencias sociales (6a reimpresión). México: FCE.

Saunders, L. (2007). "Go-betweens, gofers or mediators? Exploring the role and responsabilities of research managers in policy organisations". In: L. Saunders (Org.). Educational research and policy making. Exploring the border country between research and policy. New York: Routledge.

Shinn, T. (2007). "Jerarquías de investigadores y formas de investigación”. Redes, 12(25), 119-63. 
Stacki, S. (2003). "Other perceptions: intersubjectivity in research dialogue process". In: M. Ginsburg \& J. Gorostiaga (Eds.). Limitations and possibilities of dialogue among researchers, policymakers and practitioners. London: Routledge Falmer.

Stephan, P. (2012). How economics shapes science. Cambridge: Harvard University Press.

Tello, C. \& Mainardes, J. (2012) "La posición epistemológica de los investigadores en Política Educativa: debates teóricos en torno a las perspectivas neomarxista, pluralista y posestructuralista". Archivos Analiticos de Politicas Educativas, 20(9), 1-31.

Thoening, J. C. (2004) "La actualización del conocimiento en el campo de la administración y las políticas públicas”. In: M. C. Pardo (Comp.). De la administración pública a la gobernanza. México, DF: COLMEX.

Torgerson, D. (1992). "Entre el conocimiento y la política: tres caras del análisis de políticas". In: L. Aguilar (Ed.). El estudio de las políticas públicas. México, DF: Porrúa.

Ungerleider, C. (2012). "Affairs of the smart. Will education researchers and decision-makers hook up". In: T. Fenwick \& L. Farrell (Eds.). Knowledge mobilization and educational research. Politics, languages and responsabilities. New York: Routledge.

Weiss, C. (1979) "The many meanings of research utilization". Public Administration Review, 39, 426-431.

Wessely, S. (1996). “What do we know about peer review?”. Psychological Medicine, (26), 883-886.

\footnotetext{
Jaime Moreles Vázquez

Doctor en Educación, Universidad de Guadalajara. Profesor-Investigador de la Facultad de Pedagogía de la Universidad de Colima. Miembro del Sistema Nacional de Investigadores de México, Nivel 1.
} 